\title{
Les espaces publics d'Abu Dhabi, un terrain de jeu pour une expérience pédagogique et scientifique
}

Clio Chaveneau

\section{OpenEdition}

1 Journals

Édition électronique

URL : https://journals.openedition.org/cdg/7554

DOI : $10.4000 /$ cdg. 7554

ISSN : 2107-7266

Éditeur

UMR 245 - CESSMA

Référence électronique

Clio Chaveneau, « LeS ESPACES PUblics d'ABU DHABI, UN TERRAIN DE JeU POUR UNe EXPÉRIENCE PÉDAgogique ET SCIENTIfique », Carnets de géographes [En ligne], 15 | 2021, mis en ligne le 30 avril 2021, consulté le 27 mai 2021. URL : http://journals.openedition.org/cdg/7554 ; DOI : https://doi.org/ $10.4000 /$ cdg. 7554

Ce document a été généré automatiquement le 27 mai 2021.

La revue Carnets de géographes est mise à disposition selon les termes de la Licence Creative Commons Attribution - Pas d'Utilisation Commerciale - Pas de Modification 4.0 International. 


\title{
Les espaces publics d'Abu Dhabi, un terrain de jeu pour une expérience pédagogique et scientifique
}

\author{
Clio Chaveneau
}

1 Bien qu'elle soit au cœur de notre métier d'enseignant-chercheur, l'articulation concrète de nos activités de recherche et d'enseignement n'est que trop rarement discutée dans nos publications académiques. Géographes, sociologues et anthropologues, nous formons, semestre après semestre, les étudiants à la recherche empirique au travers d'enquête collective, menée tout au long d'un semestre, voire d'une année. Pris dans notre quotidien professionnel, nous avons rarement le temps de mettre par écrit ces expériences pourtant formatrices en tant qu'enseignant et chercheur. Comme le soulignent Charles Gadéa et Charles Soulié, la réflexion pédagogique et la formation à la recherche des étudiants du supérieur est « un genre peu répandu chez les enseignants du supérieur et les principales revues disciplinaires consacrent peu d'articles aux questions d'enseignement ou de didactique de la discipline » $(2000,158)$.

2 Certains collègues ont réalisé ce travail de mise à distance - et les réflexions scientifiques et pédagogiques qui en découlent sont riches et enthousiasmantes (Chapoulie 2000; Jounin 2014; Lévy-Vroelant 2016; Chauvin 2017; Bruneau, Thin, et Venel 2019; Nicourd 2019). Accompagner les étudiants à la recherche dans le cadre d'un enseignement de sociologie permet, à la fois, de se prêter à l'exercice de la réflexivité (Quel place et rôle pour les étudiants sur ce terrain?) et de produire des savoirs sociologiques grâce au travail de collecte de données des étudiants (via les entretiens, observations ou photographies).

3 Dans la plupart des licences et masters de sociologie - et de sciences sociales plus largement - des enseignements d'initiation à la recherche empirique sont dispensés. Pourquoi, alors, ce type de publication est-il peu répandu ? Par manque de temps mais aussi d'espace de discussion, nous prenons peu le temps de "mettre à plat» nos expériences pédagogiques et de discuter, synthétiser ou comparer d'une part, les 
bonnes pratiques comme les échecs de l'enseignement des méthodes empiriques et d'autre part, les atouts et les limites de l'implication des étudiants dans nos projets de recherche. Au-delà des contraintes matérielles, Charles Gadéa et Charles Soulié expliquent la discrétion sur ces pratiques d'apprentissage du terrain par "la suprématie du théorique sur l'empirique [qui] reste ancrée dans l'inconscient académique, même à l'intérieur des disciplines empiriques. » $(2000,162)$.

Ceci est d'autant plus problématique que les chercheurs utilisent parfois (souvent ?) les informations et les données collectées par leurs étudiants dans leurs propres travaux. En note de bas de page d'un article ou d'un ouvrage, on retrouve une mention de la participation d'étudiants à l'étude - dans le cadre d'un enseignement dispensé par l'enseignant-chercheur. Si cette mention est indispensable, elle dit peu sur les avantages et les limites de l'implication des étudiants dans les projets de recherche de leur enseignant. Et elle reste très évasive sur la manière dont une recherche menée par des étudiants peut nourrir une réflexion sociologique pour un chercheur confirmé. Ainsi, comment l'objet de recherche et la problématique ont-ils été définis ? Si le projet est préexistant, comment a-t-il été intégré à l'enseignement ? Est-ce que l'enseignant a impliqué les étudiants dans la construction de l'objet, de la méthodologie, ou ceux-ci ont-ils seulement joué le rôle de « collecteur » de données de terrain?

Convaincue de la nécessité de réfléchir à nos pratiques professionnelles d'enseignantchercheur, je souhaite proposer ici une présentation réflexive de mon utilisation d'un programme de recherche dans mes enseignements et des bénéfices comme des limites de l'implication de mes étudiants au sein de cette recherche. Cette recherche collective menée dans la capitale des Emirats Arabes Unis de janvier 2018 à janvier 2020, portait sur les usages et fonctions des espaces publics d'Abu Dhabi par ses résidents. Dès sa conception, le projet avait un objectif double : scientifique et pédagogique. C'est sur ce deuxième aspect du projet que je vais revenir dans cet article. Ainsi, après une brève présentation du projet, l'article explore comment les espaces publics d'Abu Dhabi offrent un lieu de formation à la recherche idéal dans un contexte où l'enquête de terrain est délicate à mener. Ces lieux permettent de mobiliser les compétences multiples des étudiants et ainsi contribuent à enrichir la recherche comme l'enseignement des méthodes d'enquête de terrain dans nos cursus.

\section{Etudier les espaces publics d'Abu Dhabi : un projet interdisciplinaire}

6 En 2017, un an après ma prise de fonction comme professeure assistante au département de sociologie-philosophie de la Sorbonne Abu Dhabi, j'ai initié un projet de recherche interdisciplinaire avec un collègue géographe et un collègue architecte, également en poste dans des universités d'Abu Dhabi. L'idée de départ était de mettre en synergie nos approches disciplinaires d'une série de problématiques urbaines, rassemblées dans la question des espaces publics dans la capitale des Emirats Arabes Unis. En effet, alors que les développements urbain et économique des villes émiriennes, principalement la flamboyante Dubaï, ont fait l'objet d'une attention scientifique et médiatique importante (par exemple: Krane 2009; Kanna 2011; Vora 2013; Kathiravelu 2016), il y avait encore peu de travaux qui interrogeaient les pratiques quotidiennes des habitants des ces villes, à l'exception de ceux de Yasser Elshestawy (2008; 2010; 2011), Laure Assaf (2013; 2017) et Anke Reichenbach (2015). Les 
espaces publics comme les parcs, les plages, les terrains vagues ou les parkings à ciel ouvert, offrent néanmoins une entrée pertinente pour saisir les dynamiques sociales et socio-ethniques en cours et les différentes manières d'habiter la ville, dans un contexte de "super-diversité » et de fortes hiérarchies économiques et ethniques. En effet, les principales villes émiriennes sont marquées par une démographie exceptionnelle : 90\% de la population est étrangère (majoritairement du sous-continent indien). Cette diversité culturelle et socioéconomique est manifeste à tout observateur et participe à la construction de l'image d'une société fragmentée où des frontières étanches seraient mises en place entre les groupes. Le projet sur les espaces publics visait ainsi à observer les usages comme les interactions sociales prenant place dans la ville et à interroger l'image lisse et ultra-régulée d'Abu Dhabi pour faire apparaître l'existence de lieux de rencontres et de pratiques d'appropriation individuelle et collective de la ville par ses habitants.

7 Les usagers des lieux publics d'Abu Dhabi sont, à l'image de la démographie particulière des Emirats, majoritairement originaires de l'Asie du Sud (Inde, Pakistan, Bangladesh), de l'Asie du Sud-Est (Philippines) et du Moyen-Orient (Egypte, Syrie, Palestine, Liban). Nous avons pu constater que ces lieux de sociabilité et d'activités en plein air (barbecue, pique-nique mais également activités sportives), sont fortement fréquentés le weekend (vendredi et samedi) ainsi qu'en fin d'après-midis et soirées de la semaine. L'importance des espaces publics dans le quotidien des habitants est renforcée dans la mesure où les conditions d'habitation de beaucoup de résidents ne permettent pas de recevoir (logements ou chambres partagées, lieux isolés et éloignés) (Kyriazis, Chaveneau, et Dubucs 2021). En revanche, certains lieux publics dont l'accès est payant sont fréquentés par les classes moyennes et supérieures. Dans ces espaces sélectifs, les usagers émiriens et occidentaux sont beaucoup plus visibles et contribuent à définir les pratiques qui y ont cours.

8 L'équipe de recherche était composée d'Hadrien Dubucs, Apostolos Kyriazis, Clémence Montagne et moi-même, respectivement géographe, architecte, urbaniste et sociologue travaillant dans deux universités à Abu Dhabi : Abu Dhabi University (ADU) et Sorbonne University Abu Dhabi (SUAD). Nous avions également intégré dans le projet, quatre étudiantes - Ayesha Zahid, Hanu Dilip, Shafaq Qamar et Israa Mahgoub - issues de différents cursus académiques dans lesquels nous enseignions respectivement (Bachelor of Architecture à ADU et les Masters d'Applied Sociological Research et d'Urban Planning à SUAD). L'objectif principal était d'offrir la possibilité à des étudiantes prometteuses d'acquérir une expérience de recherche valorisable sur le marché du travail local ou dans la poursuite de leurs études universitaires. Nous souhaitions également avoir une méthodologie ambitieuse : observations systématiques et répétées de 14 lieux à Abu Dhabi. Ainsi, l'implication d'assistants de recherche était nécessaire pour réaliser la collecte de données dans le temps imparti ${ }^{1}$. En effet, le projet a reçu le soutien financier de l'Abu Dhabi Department of Education and Knowledge pour une durée de deux ans (2018-2019) ainsi qu'une aide à la recherche de la Sorbonne Université Abu Dhabi. Ces financements nous ont permis de rémunérer le travail des quatre étudiantes ainsi que de financer l'achat du matériel photographique. 
Figure 1. Carte des zones et lieux étudiés dans le programme de recherche

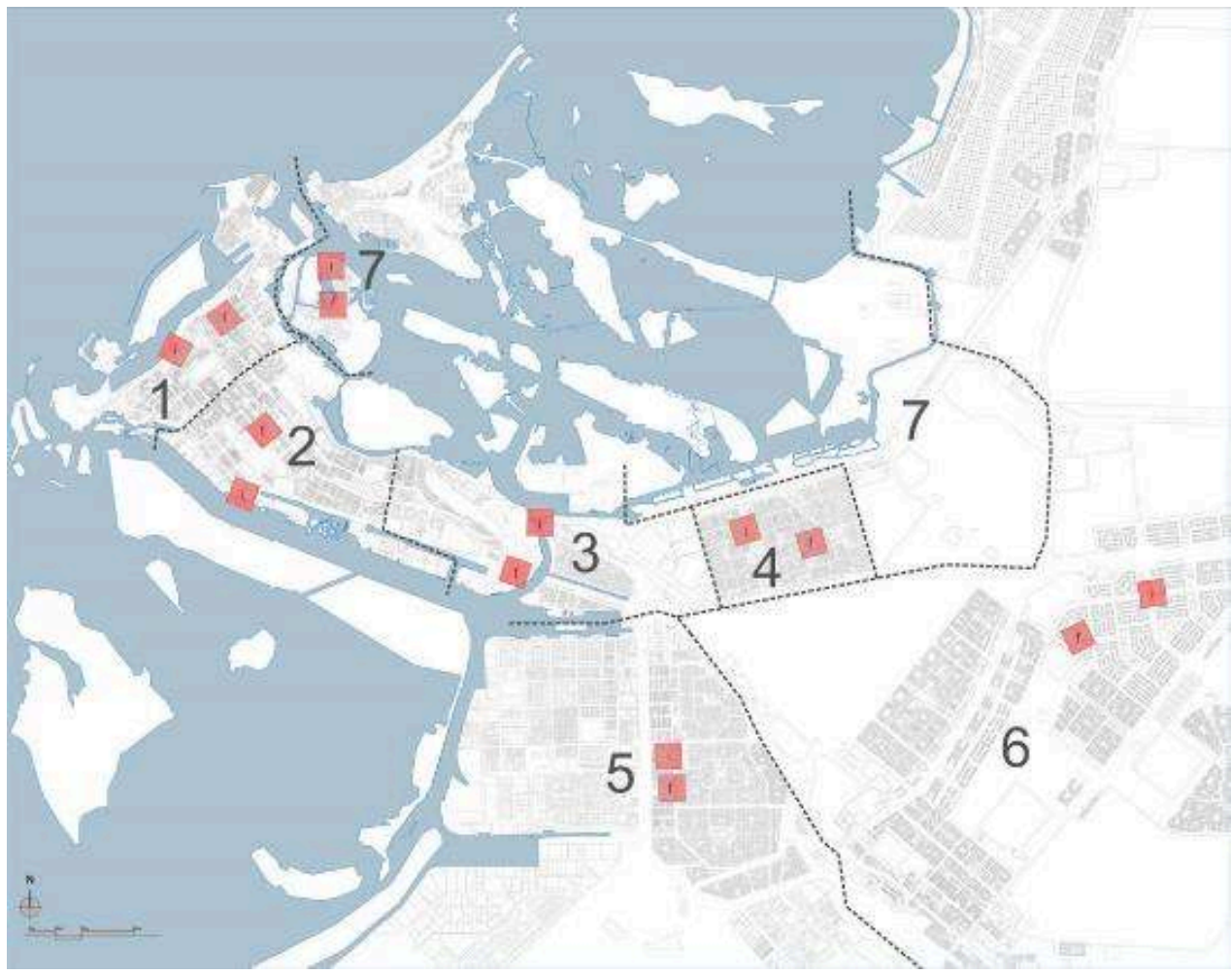

Source : Apostolos Kyriazis

9 Après avoir sélectionné 14 lieux - 7 espaces formels et 7 informels ${ }^{2}$, nous avons formé les quatre étudiantes (et notre collègue architecte) aux techniques d'observation : sur quoi doit porter leur attention, comment qualifier et définir les usagers, les pratiques et les modes d'interactions. Une grille d'observation a été préparée et nous avons effectué des sorties collectives dans quelques lieux pour «roder » la grille et s'assurer que les quatre étudiantes étaient à l'aise avec l'outil et la pratique d'observation. Ensuite, du printemps 2018 à l'hiver 2019, nous avons observé ces 14 espaces publics en adoptant le protocole suivant : six sessions d'observations comprenant trois plages horaires ( $8 \mathrm{~h}$ à 13h, 13h à $19 \mathrm{~h}$ et $19 \mathrm{~h}$ à minuit) un jour de semaine et le vendredi, seul jour chômé aux Emirats. Cela a permis de produire 84 rapports d'observation et 14 cartes d'observation qui ont ensuite été traités au travers d'un tableau d'analyse et de sous-rapports thématiques par les chercheurs du projet. 
Figure 2. Extraits d'une grille d'observation remplie par une étudiante impliquée dans le projet

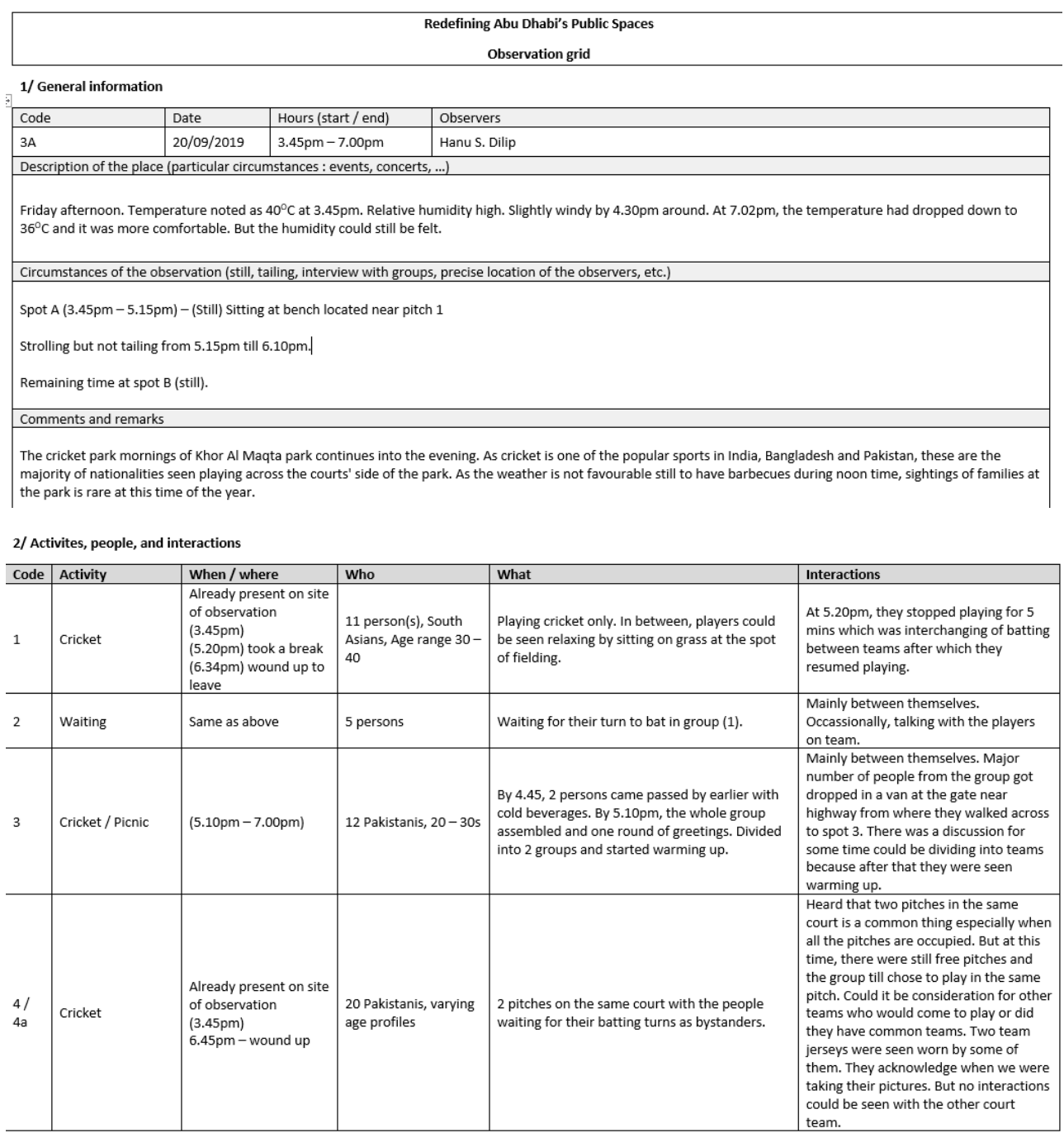

Source : Hanu Dilip

\section{De la difficulté de l'enquête de terrain aux Emirats Arabes Unis}

10 Faire de la recherche en sciences sociales aux Emirats Arabes Unis nécessite de s'adapter aux normes et règles légales et officieuses qui prévalent localement. Poser des questions à des inconnus, observer, compter, discuter de manière informelle avec des passants sont des modes de collecte de données relativement banales dans beaucoup de contextes. Mais aux Emirats, ce type de comportement se confronte à deux problèmes : la suspicion des agents de maintien de l'ordre présents dans les espaces publics et la méfiance des usagers de ces mêmes lieux. La majorité des espaces publics est soumis à une surveillance plus ou moins visible, au travers d'une part, des caméras de surveillance massivement installées dans la ville et d'autre part, des agents de sécurité surveillant dans les espaces privés comme publics, intérieurs (centres commerciaux, magasins, universités, etc.) comme extérieurs (parcs, plages, promenades, etc.) (Kyriazis, Chaveneau, et Dubucs 2021). 
11 Ainsi, l'enquêteur de terrain se trouve confronté à ces mécanismes de contrôle de l'espace et des interactions sociales. Car il passe «trop » de temps seul dans un lieu, en prenant des photographies ou des notes sur un carnet, il peut vite être considéré comme ayant une pratique suspecte ou non autorisée. Les agents demandent en général de montrer une autorisation officielle, et celle-ci est très difficile voire impossible à obtenir dans un contexte où la recherche en sciences sociales est peu comprise et fait souvent l'objet de suspicions. Mais, l'enquêteur est également confronté à la méfiance des potentiels enquêtés, qui sont rencontrés de manière impromptue dans les espaces publics. La nature des questions - centrées sur les usages - suscite une inquiétude et les échanges sont souvent brefs. L'adaptation à ce contexte est donc indispensable et nous avons dû renoncer à certaines intentions méthodologiques (court entretien informel avec les usagers) et nous centrer uniquement sur une observation discrète des lieux pour collecter des données empiriques.

Bien que conscient de ces difficultés, notre programme de recherche avait mis au cœur de son dispositif l'outil photographique. Méthode de collecte d'information et support visuel pour restituer des observations à un public extérieur, la photographie nous semblait pertinente et pédagogique pour travailler sur les espaces publics urbains. Mais la prise de photographies dans les espaces publics aux Emirats Arabes Unis est également soumise à d'importantes restrictions légales et administratives. Dans de nombreux lieux publics officiels, la photographie (autre que celle prise avec un téléphone) est souvent interdite et les agents de sécurité viennent rapidement questionner quiconque est muni d'un appareil photographique. L'appui institutionnel à notre programme, par la Sorbonne Abu Dhabi et par l'ADEK, était une condition sine qua none à cet usage afin de protéger les chercheurs et étudiants impliqués dans le projet. Nous avons donc obtenu une lettre officielle, rédigée en arabe et en anglais que les étudiantes devaient avoir sur elles lors des sessions d'observation. Toutefois, nous devions progresser dans notre enquête avec précaution et porter une attention aiguë à la discrétion et à la protection de la vie privée des observés en mettant en place des stratégies photographiques : prises de vue lointaines, prendre les usagers quand ils sont de dos, time-lapse, contre-jour volontaire. 
Figure 3. Famille émirienne se promenant dans le parc Umm El Emarat. Photographiée de dos pour préserver l'anonymat

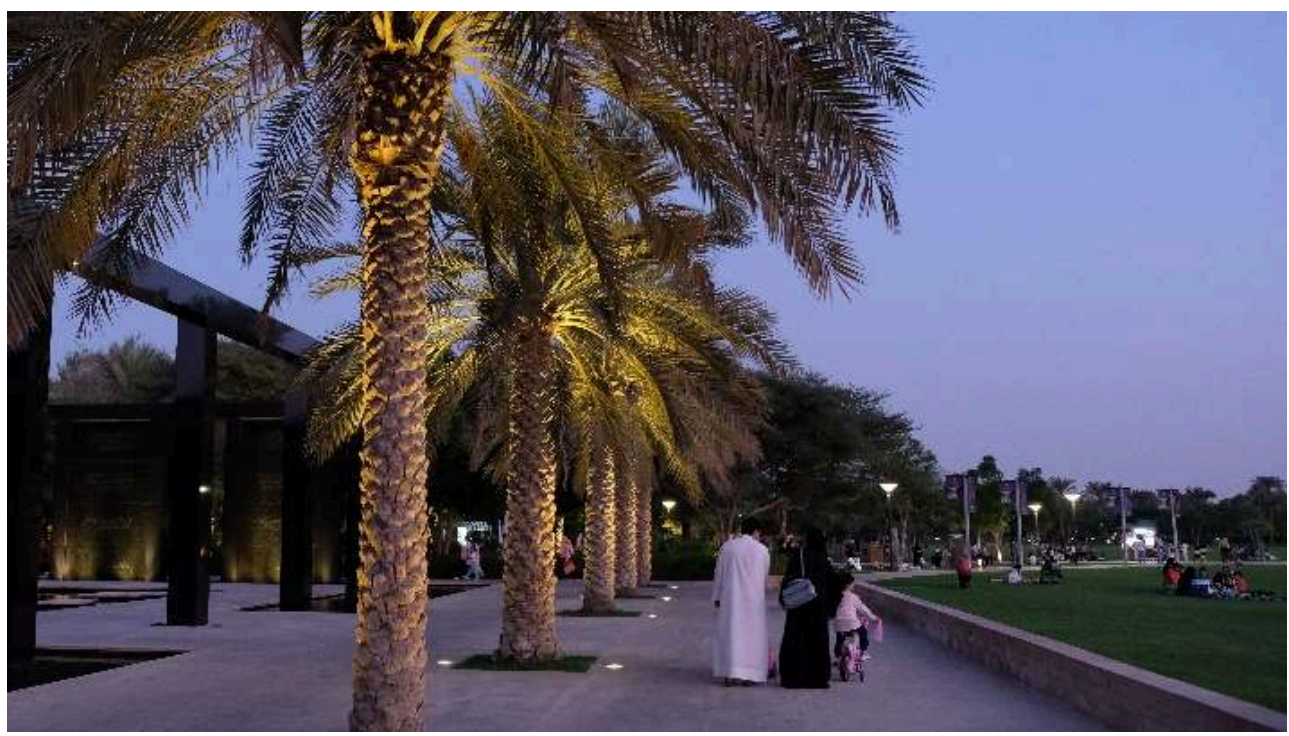

Source : Clio Chaveneau, Octobre 2020

\section{Les espaces publics : lieux d'enquête, lieux de formation}

La Sorbonne Université Abu Dhabi (SUAD) a été fondée en 2006 à la suite d'un accord entre Sorbonne Université à Paris et le gouvernement d'Abu Dhabi. Si elle est placée sous l'autorité administrative du Département de l'Éducation et du Savoir du ministère de l'Éducation émirien, SUAD est sous l'autorité académique de Sorbonne Université à Paris. À l'image de la démographie aux Emirats, la population étudiante à SUAD est très diverse, avec plus 90 nationalités présentes dans les différentes formations en sciences, droit, humanités et sciences sociales. Les enseignements ont lieu en français au niveau Licence ${ }^{3}$ - en formation initiale - et en anglais au niveau Master, tous dispensés formation continue. Plusieurs formations aux sciences humaines et sociales sont dispensées depuis sa création au sein de plusieurs départements (histoire de l'art et archéologie, histoire, géographie, philosophie et sociologie) par des Maîtres de conférences et Professeurs d'université de la Sorbonne Université, venant enseigner dans le cadre de missions courtes (en règle générale: 40h de cours, pendant deux semaines) ainsi que des enseignants dits permanents (statuts d'Assistant ou Associate Professors), résidants sur place pour au minimum trois ans.

Dans le cadre des formations dispensées dans les départements de PhilosophieSociologie et de Géographie-Aménagement, on retrouve plusieurs enseignements spécialement dédiés aux méthodes d'enquête, mais également des cours thématiques qui donnent l'occasion de mettre en pratique ces méthodes (par exemple: sociologie des migrations en L3 ou sociologie urbaine en M1). Toutefois, les lieux pour « mettre en pratique " sont relativement limités à Abu Dhabi, y compris pour des raisons climatiques (d'avril à fin octobre, les températures en journée sont comprises entre 30 et $45^{\circ} \mathrm{C}$ ). Chaque enseignant cherche et exploite, malgré tout, les différentes possibilités et temporalités qu'offre la ville pour aider les étudiants à «pratiquer les méthodes 
d'enquête ». Pierre-Marie Chauvin, maître de conférences en sociologie à Sorbonne Université a ainsi fait travailler des étudiants de la licence de Philosophie-Sociologie de la Sorbonne Université Abu Dhabi sur le quartier populaire de Mussafah à Abu Dhabi (2017). Hadrien Dubucs et moi utilisons également la ville comme lieu de mise en pratique, il nous a semblé pertinent d'articuler nos différents cours au sein d'un cadre scientifique et empirique commun. Ainsi, en plus du groupe de chercheurs et d'assistants de recherche au cœur du projet, nous avons utilisé le programme pour enseigner les méthodes d'enquête aux étudiants de premiers et seconds cycles (L1, L3 et M1) entre 2017 et 2020.

15 Les étudiants en première année des licences en Philosophie et Sociologie et en Géographie et Aménagement - une dizaine d'étudiants par classe - ont été initiés à la recherche par observation dans le cadre des cours d'introduction à la sociologie ( $5 \mathrm{~h}$ sur $39 \mathrm{~h}$ de cours, S1, enseigné par l'auteure) et d'introduction à la géographie humaine (enseigné par Hadrien Dubucs). Après leur avoir présenté et expliqué la grille d'observation conçue dans le cadre du programme, nous avons emmené deux classes d'étudiants de L1 en octobre 2018 et 2019, afin de leur permettre d'utiliser cet outil méthodologique sur plusieurs lieux étudiés. En autonomie par groupe de deux ou trois étudiants, ils avaient plusieurs heures pour collecter des informations sur l'utilisation du lieu, les usagers et faire un relevé topographique de l'espace.

Figure 4. Formation au terrain par groupe. Ici, trois étudiants de L1 géographie lors de la séance d'observation de la zone "Crown Plaza" dans le centre d'Abu Dhabi

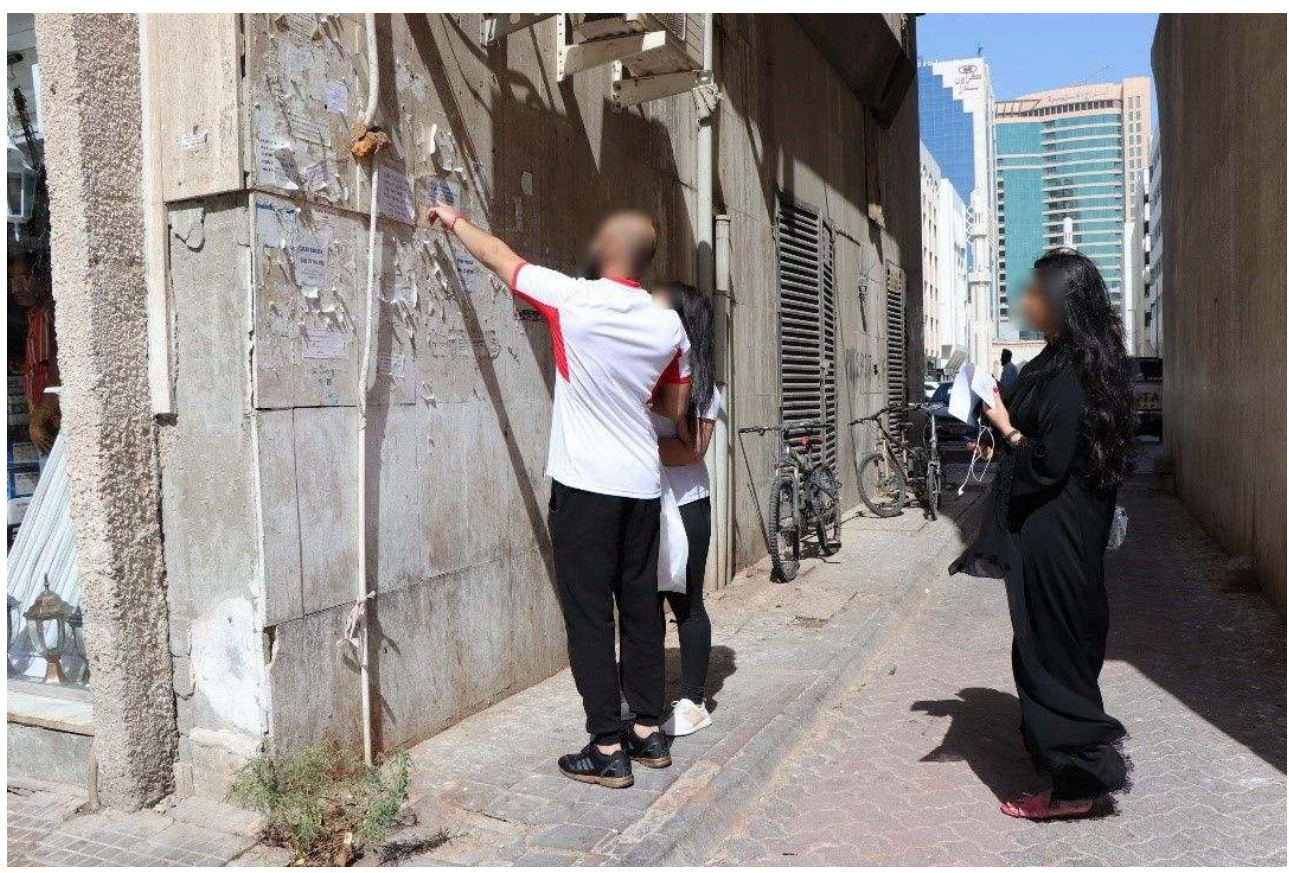

Source : Hadrien Dubucs, Novembre 2018.

Ils étaient également invités à prendre des photographies pour saisir les caractéristiques urbaines et les usages sociaux du lieu. Ces sessions d'apprentissage méthodologique, qui se sont révélées particulièrement bien accueillies, représentaient l'opportunité d'être impliqué dans un processus de recherche en cours et ainsi de mieux comprendre les étapes et les défis qui se posent au chercheur, mais également de faire l'expérience personnelle de la satisfaction ressentie à produire de la connaissance 
et à contribuer à un projet de recherche collectif et interdisciplinaire. Un usage similaire du programme de recherche a été fait dans le cadre de cours de formation aux méthodes de recherche dans deux Master (Applied Sociological Research en 2017 et Urban Planning and Development en 2019).

Figure 5. Photographies prises par une étudiante de L1 dans le quartier « Crowne Plaza » dans le centre d'Abu Dhabi : terrain vague sous un arbre

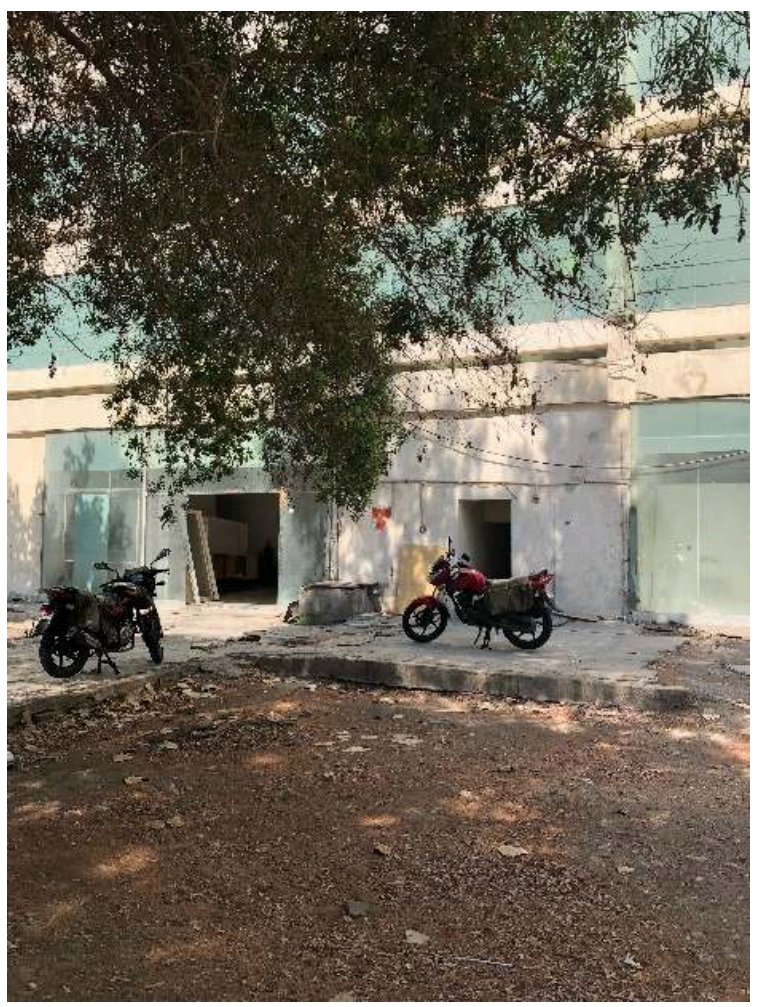

SOURCE : RANA HEMADI, NOVEMBRE 2018. 
Figure 6. Photographies prises par une étudiante de L1 dans le quartier « Crowne Plaza » dans le centre d'Abu Dhabi : voitures garées sur le trottoir

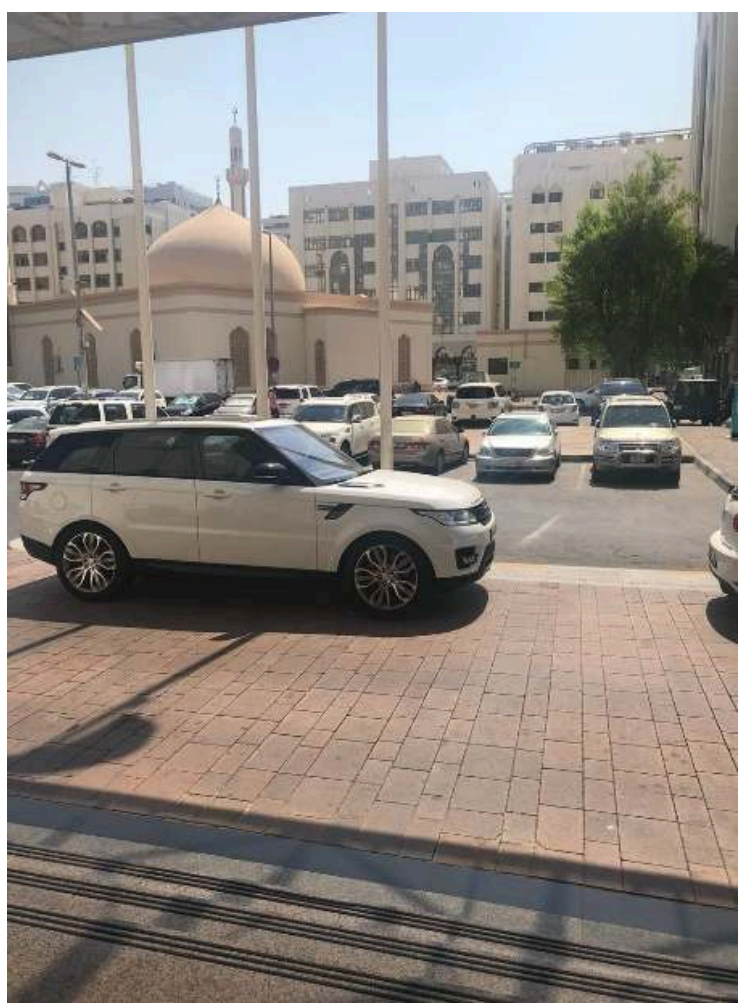

SOURCE : SHAMMA AL MEHEIRI, NOVEMBRE 2018.

En 2019, j'ai proposé un travail tout au long d'un semestre avec les étudiants de première année du Master de sociologie appliquée de SUAD (26h de cours, rythme bihebdomadaire). Divisés en deux groupes, les étudiants ont dû concevoir un projet de recherche à partir de ce thème général pour ensuite choisir une question de recherche plus précise, préparer un état de l'art sur celle-ci, choisir un lieu d'enquête parmi les espaces publics d'Abu Dhabi, concevoir leur propre grille ou un guide d'observation et enfin réaliser deux sessions d'observation (la première supervisée par l'enseignant, l'autre en autonomie) et les analyser. Le premier groupe a choisi de travailler sur les interactions sociales entre enfants et entre adultes dans les aires de jeu d'un parc très fréquenté d'Abu Dhabi et le second s'est concentré sur les usagers d'une portion précise de la Corniche d'Abu Dhabi où l'on retrouve différentes activités à côté les unes des autres (pistes de course/vélo, promenade pédestre, plage, terrasses de cafés, location de karts). 
Figure 7. Guide d'observation (en anglais) préparé par deux étudiantes de M1

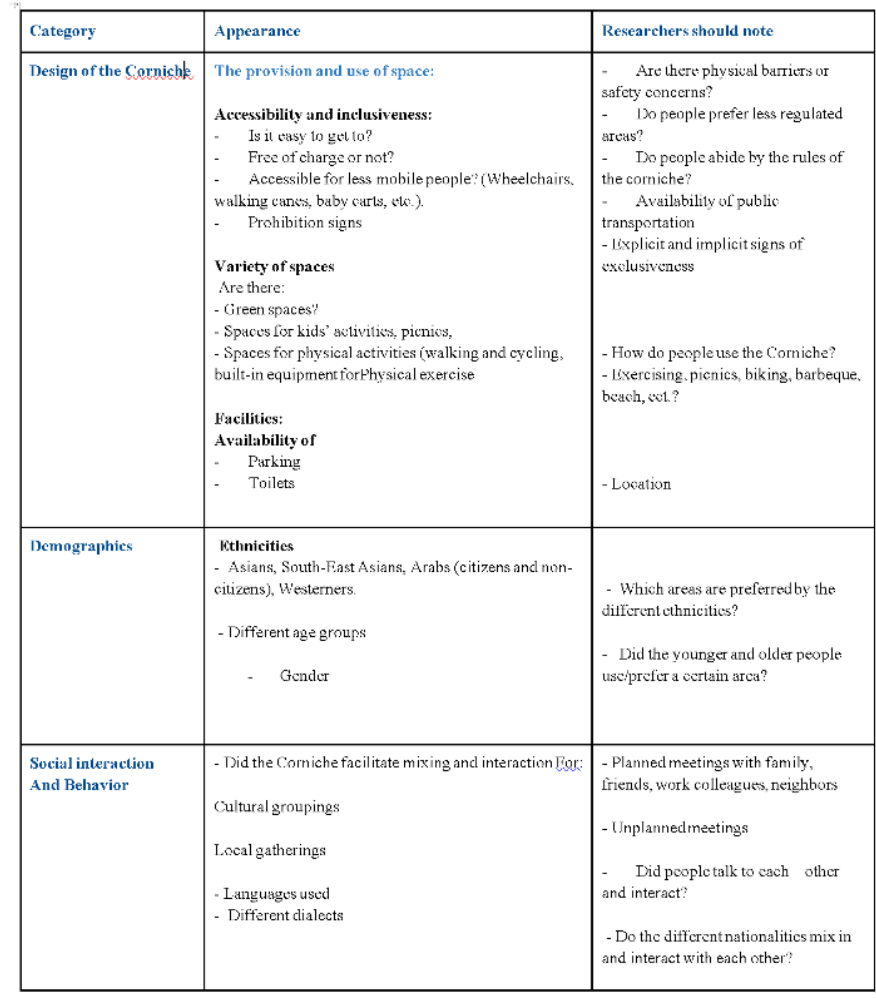

SOURCE : THURAYA SULEIMAN ET NICOLE DARROUgH.

Ainsi, les lieux étudiés dans le projet ont servi de lieux de formation pour nos étudiants. Connaissant les caractéristiques sociales et urbaines de ces lieux, nous savions qu'ils offraient un terrain de jeu sociologique pour pratiquer les méthodes d'enquête enseignées, à savoir l'observation et le recueil d'informations topographiques. Volontairement, nous avons choisi des lieux complexes, où pratiques de loisirs, marchandes et religieuses se côtoient et créent une morphologie sociale et un rythme temporel singuliers. Les restitutions en cours par les étudiants dans le cadre de dossiers ou d'exposés en groupe ont permis de mettre en avant les bénéfices de cette méthode d'enquête dans l'analyse de phénomènes urbains. Grâce à l'enquête collective, les étudiants ont pu saisir d'une part, l'utilité d'outils comme le guide ou la grille d'observation et d'autre part, la richesse des matériaux collectés grâce à l'observation d'un lieu.

L'extrait si dessous d'un des dossiers révèle bien l'intérêt méthodologique de ce type d'exercice :

«Une unique visite ne semblait pas suffisante pour obtenir assez de connaissances sur la Corniche. En raison de cette limite, une nouvelle visite de la Corniche pour une session d'observation était nécessaire pour améliorer notre compréhension de la nature de la Corniche comme un espace public inclusif. En suivant le guide d'observation préparé, la Corniche a été revisitée à trois reprises, durant les heures de pointe de trois weekends (18h30-22h), et rapidement les réveillons de Noël et du Nouvel An. En revisitant à de multiples reprises, cela nous a permis de confirmer certaines données récoltées lors de la première visite, ainsi que d'observer des différences entre les usages en semaine et durant le weekend. »

(Extrait du rapport d'enquête soumis par Thuraya Suleiman et Nicole Darrough (M1) - traduit de l'anglais par l'auteure) 

aux étudiants - y compris en Master - à mettre à distance le réel, objectiver leur quotidien pour y voir des faits sociaux et urbains. Parmi les retours formulés par les étudiants, c'est cet apprentissage du « regard sociologique» qui revient le plus. Ils soulignent leur capacité nouvelle à observer et à questionner leur environnement quotidien : Qui utilise l'espace? Qui en est privé? Comment se manifestent les hiérarchies socioéconomiques dans l'espace urbain? sont autant de questions que se sont posées nos apprentis sociologues et géographes. Ainsi, si ce n'était pas forcément envisagé comme un objectif premier, il ressort que le développement de la capacité à voir «le social» dans leur quotidien et leur environnement familier est un des bénéfices principaux du travail par projet collectif guidé par l'enseignant - au sein d'une classe d'étudiants en sociologie ou géographie humaine, y compris le temps de quelques séances de cours.

\section{Mobiliser les compétences multiples des étudiants- enquêteurs}

Au-delà du projet pédagogique, mobiliser des étudiants dans un projet de recherche permet de pallier certaines difficultés du terrain. Les méthodes d'enquête empirique peuvent être particulièrement chronophages pour des enseignants-chercheurs dont les emplois du temps sont déjà bien chargés. Dans le cadre de notre projet, où 74 sessions d'observation étaient planifiées, la participation d'assistants de recherche et l'inclusion du projet dans certains enseignements étaient judicieux - voire nécessaires. Toutefois, dans des classes très hétérogènes, on est confronté aux disparités de niveaux et de motivation des étudiants : Comment négocier ces différences au sein d'une même classe ? Quelles en sont les incidences sur le travail produit? Afin de pallier les problèmes de qualité et de rigueur empirique, nous avons mis l'accent sur la formation à l'observation - en amont des sessions de terrain. Néanmoins, la qualité des observations récoltées reste variable selon les étudiants et impose des limites dans l'utilisation que nous pouvons faire de certaines données - ou la nécessité de compléter, renforcer le travail fait par les étudiants.

Ceci dit, si des limites existent, nous avons pu également constater que les étudiants possèdent des compétences multiples sur le terrain qu'ils mobilisent volontiers. Claire Lévy-Vroelant, dans un article consacré à l'enquête menée dans les bains-douches municipaux parisiens par ses étudiants de Licence de sociologie de l'université de Paris 8 , souligne à plusieurs reprises les ressources privilégiées que possèdent ses étudiants : 
leur capacité à "se mouiller » est particulièrement frappante (Lévy-Vroelant, 2016). J’ai également pu constater la facilité avec laquelle les étudiants «entrent» dans le terrain: lors de sorties sur les espaces étudiés, ils prenaient des photos avec leur téléphone, discutaient avec des passants, interrogeaient des commerçants et employés ou encore suivaient des usagers dans leur déambulation, avec une étonnante facilité. Comme le souligne Gadéa et Soulié, ce type de sorties permet de découvrir les multiples compétences individuelles au sein d'un groupe d'étudiants qui peuvent «mobiliser des dispositions pratiques, ou " profanes ", habituellement négligées ou laissées en friche par le système scolaire, et qui s'avèrent pourtant fort utiles dans une discipline empirique comme la sociologie » $(2000,168)$. Si cela permet aux étudiants de prendre confiance en leurs capacités d'apprenti sociologue, leur spontanéité et l'engagement représentent également une véritable ressource pour une enquête collective où des chercheurs confirmés pourraient avoir tendance à prendre davantage de précaution. Nous avons pu observer comment leur jeunesse, entrain et leur statut d'étudiant participent à détendre l'atmosphère et éveillent moins de défiance auprès des usagers. Par exemple, deux étudiantes ont discuté avec des usagers de la Corniche d'Abu Dhabi et récolté des informations sur leur lieu de résidence et leur usage de ce lieu (fréquence, fréquentation, motivations pour ce lieu spécifiquement). Nos étudiants montrent une aptitude à l'adaptation et sont généralement confiants lors de la rencontre à l'autre. Leur proximité sociale ou générationnelle avec certains usagers facilite d'autant plus les échanges, comme dans l'extrait d'un compte rendu de recherche :

« Le soir de Noël, il y avait un groupe hétérogène de personnes fêtant Noel (observé autour de 21h). Des personnes de différentes ethnicités portaient des chapeaux du Père Noel et mangeaient des desserts avec leurs familles. Deux adolescents déguisés en Père Noel et tenant une cloche, étaient arrêtés par des enfants et des familles pour prendre des photos avec eux. Quand nous les avons interrogés, ils nous ont dit qu'ils étaient des lycéens et qu'ils souhaitent juste faire plaisir, rendre les gens et les enfants heureux. Ils nous ont dit que la Corniche était le meilleur endroit pour cela car la météo est agréable et qu'ils voulaient être dans un lieu public moins commercial. Ils ont indiqué que l'on retrouvait des Pères Noëls dans les centres commerciaux de la ville, alors que sur la Corniche, il y aurait sûrement plus de gens « seuls ", isolés. Lorsque l'on réfléchit à cet entretien qualitatif, l'affirmation sur les gens seuls est une indication du niveau de conscience social concernant la variété des niveaux de vie et situations économiques des usagers de la Corniche. En effet, à Abu Dhabi beaucoup de travailleurs avec de bas salaires sont séparés de leurs familles, restées dans leur pays d'origine ».

(Extrait du rapport d'enquête soumis par Thuraya Suleiman et Nicole Darrough traduit de l'anglais par l'auteure)

24 Le genre de nos étudiants - majoritairement des femmes (70-80\% dans nos filières à SUAD) - contribue peut-être également au bon accueil qui leur est fait lorsqu'elles interpellent des passants, amorcent une conversation avec des familles réunies pour un pique-nique ou des groupes d'adolescents en pleine discussion. En classe, j'ai discuté de l'utilité de penser les effets des identités sociales sur les rapports enquêteurs-enquêtés et l'accès au terrain, tant dans ses bénéfices (accès à certaines catégories, certains lieux) que ses limites. Pierre-Marie Chauvin souligne la nécessité de prendre en compte "les paramètres intersectionnels opposant potentiellement enquêteurs et enquêtés " (2017) : si le genre en est un, la situation socioéconomique (milieux privilégiés) et l'appartenance nationale ou ethnique doivent être aussi reconnues par les étudiants. J'ai ainsi fait réfléchir mes étudiants de Master - majoritairement émiriens (7 sur 10 
dans la classe) aux rapports de domination entre citoyens et résidents étrangers (et notamment les moins privilégiés) qui peuvent affecter la relation d'enquête : méfiance et évitement dans l'espace public. Certains étudiants, notamment masculins, ont ainsi fait le choix de ne pas porter la kandura ou le ghotra ${ }^{5}$ lors des séances d'observation.

Bien que nos étudiants viennent en majorité de milieux sociaux relativement favorisés aux Emirats Arabes Unis, la diversité ethnique et culturelle est néanmoins forte dans les classes. Bilingues et souvent trilingues, ils possèdent des compétences linguistiques multiples, qui peuvent justement faire défaut aux chercheurs étrangers. Nombreux sont ceux de la deuxième génération ${ }^{6}$, nés et ayant grandi aux Emirats de parents étrangers. Comprenant le malayalam, l'urdu, le tagalog, l'arabe levantin ou du golfe ou bien l'hindi, ils sont à même de mieux identifier les langues et les ethnicités présentes dans les espaces observés ainsi que de converser avec les usagers. Ces compétences multiples sont de véritables atouts sur un terrain aussi multiculturel et délicat à aborder que sont les espaces publics émiriens. Les quatre assistantes de recherche sur le programme sont Pakistanaises, Indienne et Egyptienne et ont grandi à Abu Dhabi. Les classes de L1, L3 et M1 avec qui l'on a travaillé comptent aussi des profils similaires (Marocains, Libanais, Palestiniens, Français), mais également beaucoup d'émiriens, notamment au niveau Master. Ainsi, leurs compétences et expériences de la ville spécifiques - à leur âge et leur milieu d'origine - permettent d'enrichir notre compréhension des rôles et usages des espaces publics par une population locale très hétérogène. Comme le souligne Claire Lévy-Vroelant, "ni vraiment indigènes, ni encore sociologues, mais un peu les deux à la fois, les étudiants sur le terrain opèrent une triangulation» $(2016,132)$. Cette triangulation, si elle est bien utilisée par les chercheurs confirmés peut permettre de faire émerger des résultats de recherche pertinents et de qualité.

Faire «faire du terrain » aux étudiants permet également d'observer les étudiants en train d'observer. Eux-mêmes résidents de la ville et utilisateurs des espaces publics, ils font émerger dans les discussions de préparation aux terrains mais également dans leurs compte-rendu, les représentations et pratiques qu'ils ont vis-à-vis de certains espaces publics. Par exemple, certaines étudiantes émiriennes m'ont ainsi indiqué ne pas vouloir se rendre qu'au parc Umm El Emarat. Celui-ci est en effet très apprécié des Emiriens pour la qualité des infrastructures et des services disponibles et dont l'entrée est payante - faisant office de filtre socioéconomique. Cette résistance à se rendre dans certains lieux a suscité à une discussion riche dans la classe me permettant ainsi de mieux saisir les enjeux de réputation et d'aménagement liés à certains parcs - et auprès de certaines catégories d'habitants. Ainsi, en observant nos étudiants, nous pouvons avoir accès à des manières de voir et de penser la ville en prenant en compte les effets de la stratification socioéconomique sur les pratiques.

\section{De l'impératif de valoriser le travail étudiant}

Ces expériences d'enquête collective avec le public étudiant de la Sorbonne Université Abu Dhabi m'ont permis d'affiner mon approche pédagogique de l'enseignement des méthodes d'enquête qualitatives. Au niveau licence, il est apparu qu'un cadrage très précis des étudiants est nécessaire pour les guider au mieux dans l'utilisation d'un guide d'entretien ou d'observation. Le terrain doit être bien préparé à l'avance tant d'un point de vue technique (que doit-on observer, comment aborder les passants, que 
peut-on prendre en photo, etc.) que sociologique. Au niveau Master, le travail sur un projet de recherche collectif tout au long d'un semestre - voire d'une année - est une très bonne manière de faire mettre en pratique les méthodes d'enquête, mais aussi, de préparer leur futur mémoire en leur permettant de passer par toutes les étapes de la recherche (du choix de la question de recherche jusqu'à l'analyse des données collectées). De plus, inscrire les étudiants dans un programme de recherche en cours permet de donner vie à nos disciplines et aide à faire le lien entre le monde académique et celui de la recherche.

Ainsi, cette expérience pédagogique et scientifique m’a permis de réfléchir à la place que l'on offre aux étudiants dans le cadre de nos recherches. Mettre en valeur les observations, les photographies et les découvertes des étudiants est essentiel. Mais il me semble crucial de penser plus largement les modes de valorisation des travaux de recherche étudiants. Différents outils et moyens sont à disposition et doivent être saisis : la tenue d'un blog de recherche, des restitutions orales en cours, mais également lors de séances ouvertes à l'université (en collaboration avec d'autres formations ou départements par exemple) ou encore en envisageant l'écriture d'articles à publier dans des revues étudiantes ou la corédaction d'articles scientifiques avec les étudiants les plus impliqués. Dans le cadre de notre projet, un article a été publié avec les étudiantes/assistantes de recherche comme co-auteures (Kyriazis et al. 2019). Mais il faudrait pour cela former les étudiants, dès le M1, à l'écriture d'articles scientifiques et créer davantage d'espaces de diffusion orale et écrite.

Si les avantages pour les étudiants semblent nombreux, ils le sont également pour les enseignants et chercheurs. Comme le rappellent Charles Gaéda et Charles Soulié, "l'idée que la force de travail des étudiants puisse être mise à profit par les enseignants n'est pas nouvelle. De longue date, des étudiants ont servi de "petites mains ", main d'œuvre gratuite ou presque, pour les enquêtes de terrain, ou de réservoir secret d'idées de recherche » $(2000,171)$. Les compétences et les connaissances des étudiants sont à mobiliser tout comme ils représentent aussi une entrée sur des mondes sociaux et spatiaux parfois difficilement accessibles. C'est particulièrement le cas dans le contexte des Emirats Arabes Unis, où la population est hétérogène et la société relativement hiérarchisée et stratifiée. Cette mobilisation des compétences des étudiants ne doit pas être réalisée à leurs dépens, mais dans le cadre d'une collaboration pédagogique et scientifique qui est bénéfique pour les chercheurs confirmés comme pour les apprentis chercheurs.

\section{BIBLIOGRAPHIE}

ASSAF L. (2013), « La Corniche d'Abu Dhabi : espace public et intimités à ciel ouvert », Arabian Humanities, no. 2. https://doi.org/10.4000/cy.2625.

ASSAF L. (2017), Jeunesses arabes d'Abou Dhabi (Émirats arabes unis) : catégories statutaires, sociabilités urbaines et modes de subjectivation, Thèse de doctorat d'anthropologie, Université Paris Ouest Nanterre La défense. 
BRUNEAU I, THIN D., VENEL N. (2019), « Une autre pédagogie de l'enquête qualitative. Ce que le stage de terrain fait à l'enseignement des méthodes ", Socio-logos. Revue de l'association française de sociologie, no. 14. https://doi.org/10.4000/socio-logos.4320.

CHAPOULIE J-M. (2000), « Enseigner le travail de terrain et l'observation : témoignage sur une expérience (1970-1985) », Geneses, no. 39, vol. 2, pp. 138-55.

CHAUVIN P-M. (2017), « Mussafah Shots. Une expérience pédagogique de sociologie visuelle dans une petite ville industrielle des Émirats ", Revue française des méthodes visuelles [En ligne] https:// rfmv.fr/numeros/1/articles/une-experience-de-sociologie-visuelle-dans-une-petite-villeindustrielle-des-emirats/.

ELSHESHTAWY Y. (2008), « Transitory sites: mapping Dubai's 'forgotten'urban spaces », International Journal of Urban and Regional Research, no. 32, vol. 4, pp. 968-88.

ELSHESHTAWY Y. (2010), « Little space, big space: Everyday urbanism in Dubai », Brown J. World Aff. 17: 53.

ELSHESHTAWY Y. (2011), « Informal Encounters: Mapping Abu Dhabi’s Urban Public Spaces », Built Environment, no. 7. https://doi.org/10.2148/benv.37.1.92.

GADEA C, SOULIE C. (2000), « Réflexions sur une expérience d'initiation à la recherche en sociologie à l'Université (1994-2000)», Geneses, no. 39, vol. 2, pp. 155-71.

JOUNIN N. (2014), Voyage de classes: des étudiants de Seine-Saint-Denis enquêtent dans les beaux quartiers, Paris, La Découverte.

KANNA A. (2011), Dubai, the city as corporation, Minneapolis, University of Minnesota Press.

KATHIRAVELU L. (2016), Migrant Dubai: Low Wage Workers and the Construction of a Global City, Palgrave Macmillan UK.

KRANE J. (2009), Dubai: The Story of the World's Fastest City, New York, Atlantic Books Ltd.

KYRIAZIS A., CHAVENEAU C., DUBUCS H. (2021), Abu Dhabi Public Spaces : Urban encounters, Social Diversity and (In)formality, Motivate Publishing.

KYRIAZIS A., DUBUCS H., CHAVENEAU C., MONTAGNE C., DILIP H., ZAHID A., QAMAR S. (2019), « Behavioral Mapping of Abu Dhabi's Public Spaces: Urban Research Photography and Cultural Clashes ». Sophia.

LEVY-VROELANT C. (2016), « Se mouiller au propre comme au figuré. De l'observation à l'ethnographie dans les bains-douches parisiens », Espaces et societes, no. 164-165, pp. 127-42.

NICOURD S. (2019), "L'enquête collective de recherche comme démarche pédagogique ", Sociologos. Revue de l'association française de sociologie, no. 14. https://doi.org/10.4000/socio-logos.4418.

REICHENBACH A. (2015), « Gazes That Matter: Young Emirati Women's Spatial Practices In Dubai », Urban Anthropology and Studies of Cultural Systems and World Economic Development, no. 44, vol. 1-2, pp. 113-95.

VORA N. (2013), Impossible citizens: Dubai's Indian diaspora, Durham/London, Duke University Press.

\section{NOTES}

1. En raison du climat aux Emirats arabes unis, les observations, en journée, des espaces extérieurs ne sont possibles et pertinentes que huit mois par an. De mai à octobre, les températures sont trop élevées et les espaces publics extérieurs déserts pendant la journée. 
2. Nous avons opéré une distinction entre les espaces publics identifiés et aménagés par les autorités locales (parcs, promenades, plages gérées par la municipalité ou un organisme semipublic) et les lieux transformés en espaces publics de manière informelle par les habitants du quartier ou de la ville (terrain vague, parking vide, promenade non aménagée, larges trottoirs, etc.).

3. L'année préparatoire en français intensif se déroule sur une ou deux années et permet aux étudiants non-francophones d'obtenir un diplôme universitaire (DU) nécessaire pour s'inscrire en Licence à Sorbonne Université Abu Dhabi.

4. Par exemple, avec l'usage de la photographie qui doit être réfléchi et discret.

5. Principaux éléments composants la tenue masculine traditionnelle dans la péninsule arabique et portés par la grande majorité des hommes émiriens en public.

6. L'obtention de la nationalité émirienne (en dehors du mariage) est quasiment impossible depuis plusieurs décennies. Ainsi, les enfants nés ici de parents étrangers, possèdent seulement la nationalité de leurs parents. Il faut toutefois noter que des évolutions législatives récentes, en janvier 2021 notamment, esquissent une ouverture à l'obtention de la citoyenneté émirienne à certaines catégories de résidents.

\section{RÉSUMÉS}

La recherche et l'enseignement sont les deux piliers de notre métier et pourtant, l'articulation concrète de ces deux missions reste peu mise en avant dans nos discussions et nos publications. J'explore dans cet article la manière dont nos recherches peuvent, d'un part, nourrir nos enseignements méthodologiques, et d'autre part, bénéficier de l'implication des étudiants et de leurs compétences propres. Je m'appuierai sur le travail mené en tant que chercheure et enseignante de sociologie dans le cadre d'un programme de recherche interdisciplinaire (géographie, architecture et sociologie) sur les espaces publics à Abu Dhabi (Emirats arabes unis, 2018-2020). Dans un contexte où l'enquête de terrain est délicate à mener, les espaces publics se sont révélés des lieux d'enquête et de formation privilégiés et où les étudiants nous offraient une entrée supplémentaire pour la compréhension des rapports sociaux à la ville.

Abstract: Research and teaching are the two pillars of our profession, however, the interconnection of both activities remains overlooked in our discussions and publications. I explore in this article the way our researches could, firstly, enrich our methodological courses and teaching methods, and, secondly, benefit from students' participation and their very own competences. Based on my experience as sociology professor and researcher in an interdisciplinary research program (geography, architecture and sociology) on public spaces in Abu Dhabi (United Arab Emirates, 2018-2020) where students were involved in the data collection. In a context where fieldwork research can be tricky, public spaces appeared to be ideal locations for survey and training and a place where students gave us an additional entry into the understanding of social interactions and various uses of the city. 
INDEX

Mots-clés : espaces publics, méthodes d'enquête, étudiants, formation au terrain, enseignantchercheur, pédagogie, émirats arabes unis

Thèmes : Carnets d'enseignements

Keywords : Public spaces, Research methods, Pedagogy, Teaching methods, United Arab Emirates

\section{AUTEUR}

\section{CLIO CHAVENEAU}

Professeure Assistante de sociologie, Sorbonne Université Abu Dhabi et chercheure associée au GEMASS.

Clio.chaveneau[at]sorbonne.ae 